\title{
Abruptio placentae y trombofilia hereditaria
}

\author{
Abruptio placentae and hereditary thrombophilia \\ Abruptio placentae e trombofilia hereditária
}

Rosario Morán Resumen: El abruptio placentae o desprendimiento prematuro de placenta normoinserta se define como el desprendimiento parcial o completo de la placenta normalmente implantada que ocurre antes del parto en embarazos mayores a 20 semanas. Entidad de elevada morbimortalidad (75\%). La pato-fisiología es multifactorial, disminución de la invasión trofoblástica de las arterias espirales, disfunción endotelial y activación anormal de la coagulación a nivel de la interface materno-fetal. Es controversial si la trombofilia hereditaria contribuye a este proceso y de ser así como lo haría. Se realiza una revisión del tema con recomendaciones de estudio y tratamiento en pacientes que tienen esta patología.

Palabras clave: abruptio placentae, trombofilia hereditaria

Abstract: Abruptio placentae or premature detachment of the normoinserted placenta is defined as partial or complete detachment of the normally implanted placenta that occurs before delivery in pregnancies greater than 20 weeks. Entity with high morbidity and mortality (75\%). The pathophysiology is multifactorial, decreased trophoblastic invasion of the spiral arteries, endothelial dysfunction and abnormal activation of coagulation at the level of the maternal-fetal interface. It is controversial whether and if hereditary thrombophilia contributes to this process. A review of the subject is carried out with study and treatment recommendations in patients who have this pathology.

Key words: abruptio placentae, hereditary thrombophilia

Resumo: O descolamento da placenta ou descolamento prematuro da placenta normoinserida é definido como o descolamento parcial ou completo da placenta normalmente implantada que ocorre antes do parto em gestações com mais de 20 semanas. Entidade com alta morbimortalidade (75\%). A fisiopatologia é multifatorial, diminuição da invasão trofoblástica das artérias espirais, disfunção endotelial e ativação anormal da coagulação ao nível da interface materno-fetal. É controverso se e se a trombofilia hereditária contribui para esse processo. É feita uma revisão do assunto com recomendações de estudo e tratamento em pacientes portadores dessa patologia.

Palavras-chave: descolamento da placenta, trombofilia hereditária 


\section{Introducción}

El abruptio placentae o desprendimiento prematuro de placenta normoinserta (DPPNI) se define como el desprendimiento parcial o completo de la placenta normalmente implantada que ocurre antes del parto en embarazos mayores a 20 semanas $^{1,2}$.

Presenta una incidencia de 1 cada 100-200 embarazos, en un $75 \%$ con elevada morbimortalidad, ya sea por causa materna, fetal o neonatal ${ }^{1,2}$. Dicha incidencia se reporta como en aumento en países como EEUU, Canadá, y algunos de Europa, por la presencia cada vez más frecuente de factores de riesgo predisponentes, si bien en un $50 \%$ puede ocurrir sin estos ${ }^{1,3}$. La edad gestacional de presentación varía considerablemente según la etiología presentándose hasta en un $60 \%$ en embarazos de pretermino ${ }^{1,3,4}$.

Clínicamente se puede manifestar por dolor abdominal intenso o patrón contráctil aumentado, hipertonía, genitorragia, frecuencia cardiaca fetal alterada, repercusión materna, entre otras. Es un cuadro grave, de rápida instalación que implica medidas inmediatas, por lo que determina elevada morbimortalidad materno-feto-neonatal ${ }^{1,3,4}$.

En su fisiopatología se encuentra que la causa inmediata del desprendimiento es la ruptura de vasos maternos en la decidua basal y la sangre acumulada divide la decidua. Las separaciones placentarias son causadas por sangrado arterial de alta presión en el área central de la placenta que disecciona la interfase placenta-decidua, determinando consecuencias clínicas importantes como repercusión materna en lo hemodinámico, coagulación intravascular diseminada, shock hipovolémico en lo fetal, hipoxia fetal intraútero y óbito fetal. A pesar de los grandes avances en investigación la etiología exacta sigue siendo desconocida.

Parecería que la gran mayoría de los desprendimientos placentarios estarían relacionados con un proceso de enfermedad crónica placentaria donde las anormalidades del desarrollo placentario que aparecen en forma temprana (desarrollo de las arterias espirales) conducen a necrosis decidual, inflamación, infarto y posterior sangrado, todos determinantes de insuficiencia placentaria. Menor proporción de los desprendimientos se relacionan con las causas mecánicas traumáticas abdominales (trauma abdominal cerrado o descompresión uterina rápida). El principal factor de riesgo es haber presentado un DPPNI en un embarazo previo siendo un $15 \%$ el riesgo de recurrencia ${ }^{3,4}$.

EI DPPNI forma parte de lo que en la literatura se conoce como complicaciones mediadas por la placenta. Estas incluyen la pre-eclampsia (PRE-E)-eclampsia, restricción del crecimiento fetal (RCF), muerte fetal tardía y como se mencionó el DPPNI.

La pato-fisiología de estas entidades es multifactorial donde se incluye una disminución de la invasión trofoblástica de las arterias espirales, disfunción endotelial y activación anormal de la coagulación a nivel de la interface materno-fetal ${ }^{5}$. Esto determina hipoperfusión úteroplacentaria que genera insuficiencia placentaria, hipóxia crónica e isquemia útero-placenta ${ }^{3,4,6}$. En forma global, estos trastornos a menudo se caracterizan por coexistir en un mismo embarazo, o uno puede ocurrir en una gesta y el otro evento en la siguiente. Se ha visto en estudios de cohorte que aquellas pacientes que tienen el antecedente en una primera gestación de RCF presentan mayor riesgo de presentar DPPNI en un embarazo posterior y en aquellas pacientes con PRE-E en el primer embarazo presentan mayor riesgo de desarrollo de DPPNI en una gesta posterior $^{3,4,6}$.

Es por esto que en embarazos posteriores a una complicación medida por la placenta se recomienda en siguientes embarazos hacer seguimiento con ecografías seriadas mensuales a partir de las 24-28 semanas hasta el parto para valorar el adecuado crecimiento fetal. Asimismo, una estrecha monitorización de desarrollo de PRE-E. La realización de monitorización electrónica fetal o perfil biofísico seriado en ausencia de anormalidad documentada no sería útil ni recomendable.

\section{Trombofilia hereditaria}

Es controversial si la trombofilia hereditaria $(\mathrm{TH})$ contribuye a este proceso y de ser así como lo haría. En cuanto a la relación existente entre el DPPNI y las TH solo aparece una débil relación en estudios de casos y controles, no así en estudios prospectivos.

En un meta-análisis que incluyó estudios prospectivos de cohorte no se observó incremento del riesgo de DPPNI en pacientes portadoras de Factor V de Leiden (FV Leiden) ni con la mutación de la protrombina G20210A7. En este sentido, un estudio de cohorte realizado en Canadá que incluyó 7343 mujeres embarazadas no seleccionadas que se siguieron prospectivamente y se 
testeo TH luego de parto se detectó $6.9 \%$ de prevalencia de TH y no se vieron diferencias en la incidencia de DPPNI entre las portadoras de TH vs la que no presentaban ${ }^{8}$.

Contrariamente, otros meta-análisis han mostrado una asociación modesta entre FV Leiden y Protrombina G20210A y las complicaciones mediadas por la placenta dentro de las cuales se incluye el DPPNI ${ }^{9-12}$. La diferencia de estos con los trabajos anteriores es que además de los estudios de cohorte se han incluido estudios de casos y controles con diferentes criterios de selección de la muestra por lo que las diferencias estadísticas encontradas podrían estar en relación a esto. Los estudios de casos y controles tienen un sesgo ya que se incluyen en general los casos más severos ${ }^{13-16}$

Lykke et al. han publicado los resultados de un gran estudio casos y controles en una cohorte danesa que incluyó 100000 embarazadas en un período de 5 años. Había 2032 mujeres con complicaciones mediadas por la placenta y 1851 sin complicaciones que tenía TH. Se observó una asociación modesta entre FV Leiden y DPPNI (OR 1.7, 95\%IC: 1.2-2.4). La mutación de la protrombina G20210A no mostró asociación ${ }^{17}$.

Hay datos muy limitados con respecto a estas complicaciones y las formas homocigotas o doble heterocigotas de FV Leiden y Protrombina G20210A, proteína S, proteína C y antitrombina.

En forma global se puede concluir que no existe o existe una débil asociación entre DPPNI y TH. Por otro lado, no se ha demostrado que el tratamiento con AAS y/o HBPM logre mejorar los resultados y por el contrario si se asocia a mayor número de complicaciones hemorrágicas ${ }^{14}$.

Es por ello que no se recomienda el estudio de TH ni su tratamiento con HBPM ni AAS en pacientes que han presentado DPPNI.

\section{Recomendaciones}

No se recomienda solicitar TH en forma sistemática en mujeres con antecedente de DPPNI (grado recomendación A).

No se recomienda realizar tratamiento con AAS o HBPM con el objetivo de mejorar los resultados obstétricos en mujeres con antecedente de DPPNI (grado de recomendación B).

\section{Bibliografía}

1- Ananth CV, Keyes KM, Hamilton A, Gissler M, Wu C, Liu S, et al. An international contrast of rates of placental abruption: an age-period-cohort analysis. PLoS One. 2015;10(5):e0125246.

2- Ruiter L, Ravelli ACJ, de Graaf IM, Mol BWJ, Pajkrt E. Incidence and recurrence rate of placental abruption: a longitudinal linked national cohort study in the Netherlands. Am. J. Obstet. Gynecol. 2015;213(4):573.e1-8.

3- Berghella V. Obstetric Evidence Based Guidelines. Florida: CRC Press; 2017.

4- Rasmussen S, Irgens LM, Dalaker K. Outcome of pregnancies subsequent to placental abruption: a risk assessment. Acta Obstet. Gynecol. Scand. 2000;79(6):496-501.

5- Hossain N, Paidas MJ. Adverse Pregnancy Outcome, the Uteroplacental Interface, and Preventive Strategies. Semin. Perinatol. 2007;31(4):208-212.

6- Rasmussen S, Irgens LM, Dalaker K. A history of placental dysfunction and risk of placental abruption. Paediatr. Perinat. Epidemiol. 1999;13(1):9-21.

7- Rodger MA, Betancourt MT, Clark P, Lindqvist PG, Dizon-Townson D, Said J, et al. The association of factor $\mathrm{V}$ leiden and prothrombin gene mutation and placenta-mediated pregnancy complications: $\mathrm{A}$ systematic review and meta-analysis of prospective cohort studies. PLoS Med. 2010;7(6):e1000292.

8- Rodger MA, Langlois NJ. Is thrombophilia associated with placenta-mediated pregnancy complications? A prospective cohort study: Reply. J. Thromb. Haemost. 2014;12(8):1378-1379.

9- Wu O, Robertson L, Twaddle S, Lowe GD, Clark P, Greaves M, et al. Screening for thrombophilia in high-risk situations: systematic review and cost-effectiveness analysis. The Thrombosis: Risk and Economic Assessment of Thrombophilia Screening (TREATS) study. Health Technol. Assess. 2006;10(11):1-110. 
10- Howley HEA, Walker M, Rodger MA. A systematic review of the association between factor V Leiden or prothrombin gene variant and intrauterine growth restriction. Am. J. Obstet. Gynecol. 2005;192(3):694708 .

11- Rey E, Kahn SR, David M, Shrier I. Thrombophilic disorders and fetal loss: a meta-analysis. Lancet (London, England). 2003;361(9361):901-8.

12- Lin J, August P. Genetic thrombophilias and preeclampsia: A meta-analysis. Obstet. Gynecol. 2005;105(1):182-192.

13- Skeith L, Rodger M. Anticoagulants to prevent recurrent placenta-mediated pregnancy complications: Is it time to put the needles away? Thromb. Res. 2017;151:S38-S42.

14- Abou-Nassar K, Carrier M, Ramsay T, Rodger MA. The association between antiphospholipid antibodies and placenta mediated complications: A systematic review and meta-analysis. Thromb. Res. 2011;128(1):77-85.

15- Liu L, Sun D. Pregnancy outcomes in patients with primary antiphospholipid syndrome: A systematic review and meta-analysis. Medicine (Baltimore). 2019;98(20):e15733.

16- Skeith L, Abou-Nassar KE, Walker M, Ramsay T, Booth R, Wen SW, et al. Are Anti-囚2 Glycoprotein 1 Antibodies Associated with Placenta-Mediated Pregnancy Complications? A Nested Case-Control Study. Am. J. Perinatol. 2018;35(11):1093-1099.

17- Lykke JA, Bare LA, Olsen J, Lagier R, Arellano AR, Tong C, et al. Thrombophilias and adverse pregnancy outcomes: results from the Danish National Birth Cohort. J. Thromb. Haemost. 2012;10(7):1320-5.

\section{Aporte de cada autor al trabajo}

Rosario Morán: Concepción y diseño del trabajo, recolección de datos, redacción y revisión crítica del manuscrito. 